\section{One Year Mortality Rates of Tracheostomy Cases: Life after the Knife}

\section{Ricardo Restrepo', Daron Kahn'1, Pankaj Patel'2, Bharat Awsare $^{1}$ and Michael Baram ${ }^{1 *}$}

${ }^{1}$ Department of Medicine, Division of Pulmonary and Critical Care, Jefferson University Hospital, Thomas Jefferson University, Philadelphia, USA

${ }^{2}$ Department of Surgery, Division of Trauma, Jefferson University Hospital, Thomas Jefferson University, Philadelphia, USA

\begin{abstract}
Background: Long-term mortality of critically ill patients who undergo tracheostomies is not well known.

Objective: To determine what percentage of patients remains alive after receiving tracheostomy after respiratory failure.

Methods: We performed a single center, retrospective analysis of patients undergoing tracheotomies from our 986 bed tertiary care, urban, teaching, university hospital. With strict adherence to HIPAA rules, one year death data was collected by cross matching social security numbers and the death master file of the National Technical Information Service updated to March 2013. Using this government sponsored website, patients who died in sites outside of our institution were identified.

Results: A total of 430 patients were identified and tracked based on their social security number. Only $74 \%$ of patients survived to discharge. There were no deaths attributable to the procedure itself such as bleeding, loss of airway, or surgical site infection. Overall survival at one year was $53 \%$. Outcomes were worse for the Medical Intensive Care Unit (MICU) subset of patients with only $46 \%$ of patients alive at one year. Neurologic Intensive Care Unit (NICU) patients did better at $59 \%$ survival. Surgical Intensive Care Unit (SICU) patients did best at $63 \%$ survival. Surprisingly, $7 \%$ of patients died within one week of tracheostomy.

Conclusion: Patients who receive a tracheostomy due to their critical illness often have poor long term survival. This mortality was not procedurally related, but appears to be a marker of underlying illness. This data is important to share with patients and family members as the decision is made to get informed consent for tracheostomy.
\end{abstract}

*Corresponding author: Michael Baram, Department of Medicine, Division of Pulmonary and Critical Care, Jefferson University Hospital, $111 \mathrm{~S} 11$ th St, \#6200, Thomas Jefferson University, Philadelphia, PA 19107, USA, Tel: +1 2159551671; Fax: 2159550830; E-mail: Michael.baram@jefferson.edu

Citation: Restrepo R, Kahn D, Patel P, Awsare B, Baram M (2015) One Year Mortality Rates of Tracheostomy Cases: Life after the Knife. J Emerg Med Trauma Surg Care 2: 006

Received: June 06, 2014; Accepted: February 24, 2015; Published: March 10 2015

\section{Keywords: Decision making; Palliative care; Tracheostomy}

\section{Abbreviations}

Trach: Tracheostomy;

HIPAA: Health Insurance Portability and Accountability Act of 1996; PHI: Privacy of Health Information;

ICU: Intensive Care Unit;

MICU: Medical Intensive Care Unit;

NICU: Neuro Intensive Care Unit;

SICU: Surgical Intensive Care Unit

\section{Introduction}

The long term outcomes of tracheostomy in a critically ill patient are unknown. Many studies and meta-analyses have been done showing possible benefits of tracheostomy [1]. The benefits are often described in short-term endpoints such as ICU mortality, length of stay, nosocomial pneumonia, and duration of mechanical ventilation $[2,3,4]$. Very little is known about the long term mortality or outcomes of patients who receive a tracheostomy because many studies use 30 -day mortality [5,6]. Information on long term outcomes is potentially important for decision making. Our goal of this study was to determine long-term survival of those who received a tracheostomy after a critical illness.

Frequently when patients receive tracheostomies and leave the Intensive Care Unit (ICU), continuity of care with the ICU team is lost, and the intensivist rarely knows what happens to these patients. Since clinical practice often varies from well controlled clinical studies $[7,8]$, there is tremendous heterogeneity in the literature about reported mortality in randomized studies [6]. Knowledge of what happens to these patients is made worse by poor follow up after their transfer to Long Term Acute Care Hospital (LTACH) for vent liberation or by re-admission to other health care institutions [9]. Even after discharge to an LTACH, complex health care issues remain [10]. While the liberation from ventilation rates have been reported by Jubran et al., our goal of this project was to determine the long term morbidity and mortality of tracheotomy patients [11]. During the project we found it was difficult to contact family members to determine a patient's functional status, morbidity or mortality. Given the high mortality of the pilot project, we decided to change our scope to measure the mortality of patients who had a tracheostomy placed in the ICU. Using the National Registry managed by the United States Federal Government, we were able to track patients based on social security numbers. The unique part of this retrospective study was using the National Registry to track outcomes even if the patient left the hospital, health system, or state. This project sought out patients who underwent tracheostomy as routine care, and who were not involved in study protocols, since this may reflect a different patient population that received different care or follow up.

\section{Methods}

\section{Patients}

This study was a single center, retrospective analysis done at our 986 bed tertiary care, urban, teaching, university hospital. Our 
university Institutional Review Board (IRB) approved this project with strict adherence to Health Insurance Portability and Accountability Act of 1996 (HIPAA) requirements [12]. A sequential list of elective tracheostomy was generated from medical database and billing information. Only patients over the age of 18 who received tracheostomy for their critical illness were included; patients with cancer, tracheomalacia, or emergency cricotomy subsequently revised to tracheostomy were excluded. Only failures to wean from the ventilator secondary to pulmonary, acute neuromuscular weakness, or decreased mental status were included. Patients for whom long-term data could not be found were not included (patients without US social security numbers).

\section{Facility}

Our 950 bed university hospital runs a closed subspecialty ICU model. Each ICU is run by intensivists with sub-specialty training (i.e., NICU is managed by Neuro-Intensivists.) Tracheostomy was performed after consultation with the Trauma/Critical Care service who was all board certified surgical. The necessity and timing of the tracheostomy was determined by the intensivist who the surgical intensive care unit. The decision to proceed with tracheostomy was performed after discussion of utility, timing and medical stability by the intensivists and traumatologists. Standard operating procedure at our institution is that ICU tracheostomies are performed as bedside procedures with an attending surgeon at the bedside [13].

\section{Data collection}

By querying an electronic medical record, we identified all patients who had a tracheostomy in the hospital between January 2008 and December 2010. With strict adherence to HIPAA rules, one year death data was collected by cross matching social security numbers and the death master file of the National Technical Information service updated March of 2013.

\section{End points}

The primary end point of the study was one year survival. Secondary end points were time to tracheostomy after mechanical ventilation was instituted, reason for ventilatory support, and ICU and hospital mortality.

\section{Results}

Our electronic medical records search identified 454 tracheostomies that were performed by the trauma service. Of these, 24 patients did not have valid US Social Security numbers to compare against the national federal registry; hence only 430 patients were able to be traced after discharge. The average patient age was 61.8 years, and $60 \%$ were male. The distributions of ICU types were: SICU 102, NICU 117, and MICU 211. The largest percentages of ICU diagnoses were: unspecified respiratory failure $23 \%$, sepsis $14 \%$, and intracranial hemorrhage $10 \%$. The remaining diagnoses are listed in table 1. Historical average APACHE II scores for the MICU on admission were $18( \pm 9.0)$ [14]. Number of days of intubation prior to tracheotomy was $10.8( \pm 6.4)$ days. The three ICU types varied in timing to tracheotomy: SICU $10.5( \pm 8.0)$ days, NICU $9.1( \pm 10.0)$ days, and MICU 11.9 ( \pm 4.8) days. The average ICU stay was $31.6( \pm 28.4)$ days with individual break downs of SICU $33.2( \pm 28.0)$, NICU 26.7 ( \pm 10.0), and MICU 33.7 ( \pm 30.5). Average hospital length of stay was $38.5( \pm 21.8)$ days with individual break downs of: SICU 45.8 ( \pm 76.8$)$, NICU $30.8( \pm 21.4)$ days, and the MICU was $39.1( \pm 26.0)$ days.

\begin{tabular}{|c|c|}
\hline $80(19 \%)$ & Primary respiratory failure- Not specified \\
\hline $60(14 \%)$ & Sepsis \\
\hline $45(10 \%)$ & Intracranial Hemorrhage $(\mathrm{ICH})$ \\
\hline $36(8 \%)$ & Spinal process \\
\hline $32(7 \%)$ & Abdominal process \\
\hline $27(6 \%)$ & Pneumonia \\
\hline $26(6 \%)$ & Cerebral vascular event \\
\hline $20(5 \%)$ & Encephalopathy \\
\hline $19(5 \%)$ & Motor Vehicle Accident (MVA) \\
\hline $15(3 \%)$ & Post-Surgery \\
\hline $14(3 \%)$ & Cardiogenic shock \\
\hline $9(2 \%)$ & Central Nervous System infection \\
\hline $9(2 \%)$ & Cardiac arrest \\
\hline $9(2 \%)$ & Cardiac arrest \\
\hline $8(2 \%)$ & Gastrointestinal Bleed \\
\hline $6(1 \%)$ & Liver failure \\
\hline $6(1 \%)$ & Bone Marrow Transplant \\
\hline $6(1 \%)$ & Aspiration Pneumonia \\
\hline $5(1 \%)$ & Pulmonary Embolism \\
\hline $4(1 \%)$ & Gun Shot Wound \\
\hline $3(1 \%)$ & Pancreatitis \\
\hline
\end{tabular}

Table 1: Diagnosis requiring intubation.

One year survival was $53 \%$. Table 2 , shows that $26 \%$ of patients died while in the hospital. Of the patients who died in the hospital, $27 \%$ died within one week of tracheotomy. Table 2, further describes the timing of the 89 deaths which occur within a year of hospital discharge. Of the one week tracheotomy deaths: the MICU had 23 patients (72\%), SICU had 6 patients (19\%) and the NICU had 3 patients (9\%). Thirteen of the 32 patients had respiratory failure as the cause of intubation, 10 patients had sepsis (not including pneumonia since these were tabulated with the pneumonia patients).

There were no tracheostomy associated deaths. No cases required antibiotics to treat local surgical infections. No cases had to go to the operating room for bleeding. No case had a lost airway requiring a re-oral intubation.

\begin{tabular}{|c|c|c|}
\hline 454 & \multicolumn{2}{|c|}{$\begin{array}{l}\text { Tracheostomies performed between January } 2008 \text { and December } 2010 \\
\text { - } 24 \text { Unable to track Social Security Number }\end{array}$} \\
\hline 430 & \multicolumn{2}{|c|}{ - 112 of $430(26 \%)$ Patients died in the hospital } \\
\hline & \multicolumn{2}{|c|}{ Timing of Hospital Death Post Tracheostomy } \\
\hline & $\begin{array}{l}\gg 31 \text { of } 112(27 \%) \\
\text { » } 54 \text { of } 112(48 \%) \\
\text { » } 27 \text { of } 112(24 \%)\end{array}$ & $\begin{array}{l}\text { Die within } 1 \text { week } \\
\text { Die within } 1-4 \text { weeks } \\
\text { Die after } 4 \text { weeks }\end{array}$ \\
\hline 318 & \multicolumn{2}{|c|}{ Patients discharged alive ( $74 \%$ of original 430 patients) } \\
\hline & \multicolumn{2}{|c|}{ Timing of Death Post Hospital Discharge } \\
\hline & $\begin{array}{l}\text { " } 2 \text { of } 89(2 \%) \\
\text { " } 11 \text { of } 89(12 \%) \\
\text { " } 37 \text { of } 89(42 \%) \\
\text { " } 18 \text { of } 89(20 \%) \\
" 21 \text { of } 89(24 \%)\end{array}$ & $\begin{array}{l}\text { Die in one week } \\
\text { Die in one month } \\
\text { Die in } 1-3 \text { months } \\
\text { Die in three to six months } \\
\text { Die in six months to one year }\end{array}$ \\
\hline 229 & \multicolumn{2}{|c|}{ Alive over 1 year ( $53 \%$ of original 430 patients) } \\
\hline
\end{tabular}

Table 2: Timing of Death from Post-Tracheostomy Placement. 


\section{Discussion}

The goal of this study was to help determine the long term mortality of tracheostomy even after hospital discharge. Understanding the long term outcomes of these patients may be potentially useful in informed decision making when considering tracheostomy. Approaching families early in the ICU course is a growing trend across the country [1]. Earlier previous studies have suggested that early tracheostomy reduces length of stay in the ICU. Due to resource allocation and cost containment efforts, earlier time to tracheotomy became a trend [15]. Some of these tracheostomies are performed to facilitate patient transfer to long term ventilator facilities. However, there were still some patients that had a prolonged hospital stay after tracheostomy was performed with a mean hospital length of stay of 39 days. Although most of the tracheostomies at our center were done at 10.8 days $( \pm 6.4)$ which is neither early nor late, hospital mortality still approached $26 \%$. This mortality was associated with the underlying disease state since there were no identifiable procedure related mortalities or unforeseen events associated with bleeding, local infection, or lost airway. The lack of complications shows that the procedure can be done in critically ill patients safely at the bedside, which is consistent with other publications [16].

The most surprising finding was the high hospital mortality. Of the 112 hospital deaths, 51 patients were converted to comfort measure and died in the hospital. The most common diagnosis of these patients was pneumonia. Respiratory failure comprised $40 \%$ of the patients, and $14 \%$ of the patients had sepsis (not including pneumonia). Despite the fact the average time to tracheotomy was $12.5( \pm 5.3)$ days, the average survival was 38.8 ( \pm 20.6 .5 ) days. Given this long survival, the need for tracheotomy is understandable since the hospital stay was longer than the traditional 21 days that is typically considered as the maximum duration of oral intubation. The patients that generated wide standard deviation were most interesting: specifically the -22.5 days sub-population. This group would be the sub-group who died early post tracheotomy. Of particular interest was this large number of patients who died in the first week post tracheostomy. The most frequent diagnoses were pulmonary related processes (pneumonia, non-specific respiratory failure and pulmonary emboli). This raises the question if there are ways to potentially avoid this procedure in these early mortality groups. The high, early mortality reflects the multiple pressures to early tracheotomy due to many factors including patient comfort, finance, disposition, and ease of weaning.

The data reported here was collected prior to the release of TracMan study [17]. Our selection of patients likely represents similar populations to the TracMan study. Our retrospective analysis revealed a broad perspective of what occurs in a large urban university hospital. The generality of this paper is not intended to be applied to individual disease states. Instead it is intended to help families understand that post-ICU respiratory failure that requires a tracheostomy carries a high mortality even after discharge from the hospital. This mortality rate can be seen in other studies. TracMan study also had a similar $20 \%$ one month mortality [17]. It would have been helpful to correlate mortality with traditional scoring systems such as Sequential Organ Failure Assessment (SOFA) or APACHE II, but this is very difficult to compare due to the retrospective nature of the study $[14,18]$. Intubation affects the verbal points of the Glasgow Coma Score needing to calculate the APACHE II score.

As there is a wide standard deviation for time to tracheotomy ( 6.4 days), there was also a wide range of time to tracheostomy amongst clinicians. Even when done by study protocols, there is still variation in time to tracheostomy. Although subgroup analyses create smaller groups, the Neuro ICU tended to perform tracheostomy earlier than medical ICUs with higher percentage one year survival. This difference can be explained by different underlying diagnosis such as stroke and intracranial hemorrhage compared to highly unpredictable sepsis mortality.

The discussion of "need for tracheotomy" is often an important time in deciding goals of care in the ICU. Families need to understand that a patient may survive the acute critical care illness, but often die during the post ICU period. Families often factor long-term outcomes into the decision. Families often have a hard time grasping the "big picture" of what their loved one will be like post tracheostomy, but they must be part of the decision making team. The shared decision making model has been the standard of care in the United States for over a decade [19]. Ideally patients can define their goals of health care depending on quality of life and personal, religious, and social values. Patients choose differently based on their own intrinsic beliefs. For example when patients with ALS decide if they want a tracheostomy to prolong life, they usually have time to weigh the risks and benefits. As the disease progresses to the point of airway compromise, different decisions are made based on individual preference [20]. In the ICU, families become surrogate decision makers. It is our role as physicians to share, to the best of our knowledge, long-term outcomes of patients needing tracheostomy. This paper addresses survival of patients who receive their tracheostomy in the setting of acute critical illness. This project did not address patients who receive tracheostomy for progressive disease or malignancy.

The shortcomings of the study are that it is a retrospective study which is prone to inherent bias of retrospective analysis. Sub-group analysis of different populations was intentionally not done. The small numbers of each diagnosis, and the lack to data to exactly define each patient and patient population would be fraught with error. The intent was to develop some background data about what happens to patients post ICU, so families can make the best decisions. Similar studies have been done in nephrology to help paint big picture ideas [21]. The high overall mortality in this ICU group suggests that tracheostomy is a marker of overall illness, similar to Percutaneous Endoscopic Gastrostomy tube (PEG). Although the complication rates are low, there are many downstream sequela that are not understood $[22,23]$.

\section{Conclusion}

While being a relatively safe procedure, tracheostomy, is associated with high mortality at one year which implies that the prolonged respiratory failure with need for tracheostomy is a marker of overall illness and comorbidity. This information is potentially important during informed decision making. Better tools are needed to identify patients at highest risk of mortality both in the short term and long term after tracheostomy.

\section{References}

1. Griffiths J, Barber VS, Morgan L, Young JD (2005) Systematic review and meta-analysis of studies of the timing of tracheostomy in adult patients undergoing artificial ventilation. BMJ 330: 1243.

2. Saffle JR, Morris SE, Edelman L (2002) Early tracheostomy does not improve outcome in burn patients. J Burn Care Rehabil 23: 431-438.

3. Peñuelas O, Frutos-Vivar F, Fernández C, Anzueto A, Epstein SK, et al. (2011) Characteristics and outcomes of ventilated patients according to time to liberation from mechanical ventilation. Am J Respir Crit Care Med 184: 430-437. 
4. Frutos-Vivar F, Esteban A, Apezteguía C, Anzueto A, Nightingale P, et al. (2005) Outcome of mechanically ventilated patients who require a tracheostomy. Crit Care Med 33: 290-298.

5. Kollef MH, Ahrens TS, Shannon W (1999) Clinical predictors and outcomes for patients requiring tracheostomy in the intensive care unit. Crit Care Med 27: $1714-1720$.

6. Gomes Silva BN, Andriolo RB, Saconato H, Atallah AN, Valente O (2015) Early versus late tracheostomy for critically ill patients. Cochrane Database Syst Rev 3: CD007271.

7. Bagga S, Paluzzi DE, Chen CY, Riggio JM, Nagaraja M, et al. (2014) Improved compliance with lower tidal volumes for initial ventilation setting- using a Computerized Clinical Decision Support System. Respir Care 59: 6.

8. Rubenfeld GD, Cooper C, Carter G, Thompson BT, Hudson LD (2004) Barriers to providing lung-protective ventilation to patients with acute lung injury. Crit Care Med 32: 1289-1293.

9. Dermot Frengley J, Sansone GR, Shakya K, Kaner RJ (2014) Prolonged mechanical ventilation in 540 seriously ill older adults: effects of increasing age on clinical outcomes and survival. J Am Geriatr Soc 62: 1-9.

10. Nasraway SA, Button GJ, Rand WM, Hudson-Jinks T, Gustafson M (2000) Survivors of catastrophic illness: outcome after direct transfer from intensive care to extended care facilities. Crit Care Med 28: 19-25.

11. Jubran A, Grant BJ, Duffner LA, Collins EG, Lanuza DM, et al. (2013) Effect of pressure support vs unassisted breathing through a tracheostomy collar on weaning duration in patients requiring prolonged mechanical ventilation: a randomized trial. JAMA 309: 671-677.

12. NIH (2004) Clinical Research and the HIPAA Privacy Rule, NIH, USA.

13. Bowen CP, Whitney LR, Truwit JD, Durbin CG, Moore MM (2001) Comparison of safety and cost of percutaneous versus surgical tracheostomy. Am Surg 67: 54-60.
14. Knaus WA, Draper EA, Wagner DP, Zimmerman JE (1985) APACHE II: a severity of disease classification system. Crit Care Med 13: 818-829.

15. Hsu CL, Chen KY, Chang CH, Jerng JS, Yu CJ, et al. (2005) Timing of tracheostomy as a determinant of weaning success in critically ill patients: a retrospective study. Crit Care 9: 46-52.

16. Zagli G, Linden M, Spina R, Bonizzoli M, Cianchi G, et al. (2010) Early tracheostomy in intensive care unit: a retrospective study of 506 cases of video-guided Ciaglia Blue Rhino tracheostomies. J Trauma 68: 367-372.

17. Young D, Harrison DA, Cuthbertson BH, Rowan K, TracMan Collaborators (2013) Effect of early vs late tracheostomy placement on survival in patients receiving mechanical ventilation: the TracMan randomized trial. JAMA 309: 2121-2129.

18. Ferreira FL, Bota DP, Bross A, Mélot C, Vincent JL (2001) Serial evaluation of the SOFA score to predict outcome in critically ill patients. JAMA 286: 17541758.

19. Elwyn G, Frosch D, Thomson R, Joseph-Williams N, Lloyd A, et al. (2012) Shared decision making: a model for clinical practice. J Gen Intern Med 27: 1361-1367.

20. Chan T, Devaiah AK (2009) Tracheostomy in palliative care. Otolaryngol Clin North Am 42: 133-141.

21. Wald R, Quinn RR, Luo J, Li P, Scales DC, et al. (2009) Chronic dialysis and death among survivors of acute kidney injury requiring dialysis. JAMA 302: $1179-1185$.

22. Li I (2002) Feeding tubes in patients with severe dementia. Am Fam Physician 65: 1605-1610, 1515.

23. Murphy LM, Lipman TO (2003) Percutaneous Endoscopic Gastrostomy Does Not Prolong Survival in Patients With Dementia. Arch Intern Med 163:13511353. 